\title{
Correction to: CIRSE Clinical Practice Manual
}

\author{
Andreas H. Mahnken ${ }^{1}$ Esther Boullosa Seoane ${ }^{2} \cdot$ Alessandro Cannavale $^{3}$. \\ Michiel W. de Haan ${ }^{4} \cdot$ Rok Dezman $^{5,6} \cdot$ Roman Kloeckner $^{7} \cdot$ Gerard O'Sullivan $^{8}$. \\ Anthony Ryan' ${ }^{9}$ Georgia Tsoumakidou ${ }^{10}$
}

Published online: 22 July 2021

(C) Springer Science+Business Media, LLC, part of Springer Nature and the Cardiovascular and Interventional Radiological Society of Europe (CIRSE) 2021

\section{Correction to: Cardiovasc Intervent Radiol https://doi.org/10.1007/s00270-021-02904-3}

The name of the third author was spelled wrong, it should be: Alessandro Cannavale.
Publisher's Note Springer Nature remains neutral with regard to jurisdictional claims in published maps and institutional affiliations.

The original article can be found online at https://doi.org/10.1007/ s00270-021-02904-3.

Andreas H. Mahnken

mahnken@med.uni-marburg.de

Esther Boullosa Seoane

esther.boullosa.seoane@ sergas.es

Alessandro Cannavale

alessandro.cannavale@hotmail.com

Michiel W. de Haan

m.de.haan@mumc.nl

Rok Dezman

rok.dezman@kclj.si

Roman Kloeckner

Roman.Kloeckner@gmail.com

Gerard O'Sullivan

lahinchman1923@gmail.com

Anthony Ryan

anthonyryanir@gmail.com

Georgia Tsoumakidou

Georgia.Tsoumakidou@chuv.ch

1 Clinic of Diagnostic and Interventional Radiology, Marburg

University Hospital, Baldingerstrasse, 35043 Marburg,

Germany
2 Department of Vascular and Interventional Radiology, University Hospital of Vigo, Vigo, Spain

3 Department of Radiological Sciences, 'Policlinico Umberto I'University Hospital, Rome, Italy

4 Department of Radiology, Maastricht University Medical Center, Maastricht, The Netherlands

5 Clinical Institute of Radiology, University Medical Centre Ljubljana, Zaloska 7, 1000 Ljubljana, Slovenia

6 Faculty of Medicine, University of Ljubljana, Vrazov trg 2, 1000 Ljubljana, Slovenia

7 Department of Diagnostic and Interventional Radiology, Johannes Gutenberg-University Medical Center, 55131 Mainz, Germany

8 U.C.H. Galway, Interventional Radiology, Galway, Ireland

9 University Hospital Waterford and Royal College of Surgeons in Ireland, Waterford, Ireland

10 University Hospital of Lausanne, Lausanne, Switzerland 